\title{
Emodialisi extracorporea domiciliare: esperienza di un Centro e prospettive future
}

\author{
Salvatore David
}

\author{
Dipartimento di Clinica Medica, Nefrologia e Scienze della Prevenzione, \\ Università di Parma, Parma
}

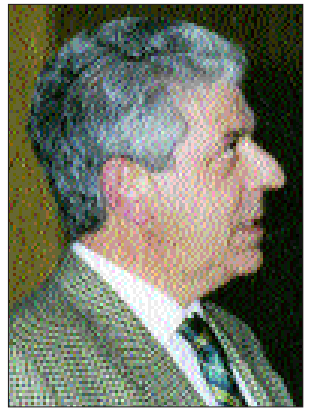

$\int$ emodialisi extracorporea domiciliare raggiunse il massimo sviluppo a Parma nella metà degli anni '70, quando circa 40 pazienti, a fronte di 80-85 trattati con la dialisi ospedaliera, erano stati avviati a questa modalità di trattamento. Questo orientamento condiviso in quel periodo da pochissimi altri Centri trova spiegazione nella coincidenza di circostanze particolari contingenti, suffragate da forti motivazioni del personale medico e infermieristico, che trovavano riscontro in analoghe positive esperienze in altri Paesi. L'esame dei fattori che portarono a quelle particolari scelte può essere utile per confrontare la realtà attuale con quel momento storico e trarre da quella esperienza informazioni utili a identificare l'eventuale spazio che il trattamento domiciliare può occupare oggi nella gamma delle terapie sostitutive artificiali .

Negli anni '70 la dialisi cominciava a uscire dalla fase pionieristica, offrendo a un numero sempre più ampio di pazienti affetti da insufficienza renale cronica la prospettiva non solo di una sopravvivenza prolungata ma soprattutto di una accettabile qualità di vita e di una valida riabilitazione socio-lavorativa. Il merito di questi risultati, andava attribuito anche alla emergente realtà della dialisi breve (4 ore tre volte la settimana o tre ore a giorni alterni, anziché le 6-8 ore tradizionali) che vedeva a Parma le prime applicazioni sotto la guida del professor Vincenzo Cambi. Di fronte alla richiesta di dialisi ampiamente superiore alle offerte, dato il numero limitato di ospedali dove questo trattamento era disponibile, la scelta della dialisi domiciliare era quasi obbligata e rappresentava spesso l'unica alternativa di vita, specie per i pazienti residenti lontano dai Centri ospedalieri attrezzati per la dialisi. I pazienti seguiti dal Centro di Parma risiedevano infatti in un territorio che aveva superato non solo i limiti provinciali, ma anche quelli regionali estendendosi dal Veneto alla Liguria e dal Trentino al Lazio. Grazie alle leggi sanitarie del tempo e, soprattutto, alla sensibilità per il problema della emergente terapia dialitica da parte dei responsabili sanitari della città, i pazienti ricevevano in uso le macchine per dialisi e venivano riforniti gratuitamente presso il loro domicilio del materiale di consumo. Il successo della dialisi domiciliare nasceva anche da un accurato addestramento al trattamento, che coinvolgeva il paziente e il partner non solo nella gestione della macchina ma anche nell'osservanza pienamente consapevole delle indicazioni dietetiche e delle prescrizioni farmacologiche. La semplicità costruttiva delle apparecchiature, prive della complicata elettronica di oggi, consentiva di rendere il paziente in grado di identificare eventuali problemi tecnici e spesso di porvi rimedio direttamente, almeno nei casi meno complicati. L'addestramento comprendeva anche la conoscenza dei sintomi principali delle complicanze cliniche dell'uremia, per un dialogo più partecipe col medico di riferimento.

Come facilmente comprensibile, le caratteristiche dei pazienti trattati erano nettamente diverse da quelle dei pazienti attuali e si confacevano ai requisiti necessari per una gestione domiciliare del trattamento. L'età media era inferiore ai quarant'anni, gli ultrasessantenni una sparuta minoranza e il diabete una rara associazione morbosa. L'alternativa del trapianto praticamente inesistente e la disponibilità della dialisi peritoneale solo come trattamento intermittente ospedaliero restringevano ulteriormente le possi- 
bili scelte terapeutiche. Sarebbe tuttavia limitativo considerare la dialisi domiciliare di quei tempi solo come l'alternativa obbligata alla morte: innegabili aspetti favorevoli, che la dialisi ospedaliera non avrebbe potuto offrire, erano il ridotto rischio di contrarre infezioni, come l'epatite virale, per la quale non esisteva ancora la vaccinazione contro il virus $B$, e una riabilitazione sicuramente migliore. Questa derivava non solo dalla maggiore libertà del paziente e dalla confortevolezza dell'ambiente familiare, ma anche dai positivi risvolti psicologici che l'attivo coinvolgimento del paziente nella cura della sua malattia comportava, favorendone l'accettazione e ripristinando la sua fiducia nella capacità di reagire alla malattia. Benché la somma di questi aspetti favorevoli superasse di gran lunga quella degli aspetti negativi collegati all'ansia che l'autogestione del trattamento poteva determinare, la dialisi domiciliare si esaurì progressivamente a Parma e non venne più rifornita di nuovi pazienti negli anni '80, fino a scomparire del tutto. La causa principale del drop-out dei pazienti trattati, a parte le complicanze cliniche che ne compromettevano l'idoneità, si può identificare nei problemi psicologici emergenti nel medio-lungo termine dalla gestione familiare del malato cronico. La dialisi domiciliare coinvolgeva infatti quasi obbligatoriamente un partner, deputato all'inserzione degli aghi nella fistola e al controllo attento della seduta dialitica. Questo atto dovuto poteva essere vissuto in maniera conflittuale dal partner, che diventava in pratica anch'esso schiavo della macchina e, in un certo senso, anche del malato. Questi riponeva spesso nel partner maggiori aspettative che in un infermiere, coinvolgendolo in un gioco di responsabilità e affettività che finiva per logorare i rapporti familiari meno solidi in breve tempo. Erano frequenti le richieste di trasferimento temporaneo del paziente al Centro, spesso clinicamente poco giustificabili e vissute quasi con senso di colpa, motivate dalla esigenza di un alleggerimento di eccessive tensioni familiari. È difficile condividere l'opinione, espressa da altri, che questa condizione rafforzasse i legami familiari: ciò era probabilmente vero nei casi di ambienti familiari molto equilibrati, ma sicuramente i rapporti familiari meno solidi finivano per incrinarsi, con inevitabile drop-out del paziente.

La nascita dei Centri ad assistenza limitata, dove il paziente partecipava attivamente alla gestione della dialisi con la sorveglianza infermieristica e senza il coinvolgimento di un familiare rappresentò la più logica e valida alternativa alla dialisi domiciliare. Le esigenze di assistenza infermieristica erano anche in aumento, a causa delle mutate condizioni cliniche dei pazienti trattati, di età sempre più avanzata e con patologie associate sempre più rilevanti. La diffusione quasi capillare dei Centri ad assistenza limitata nella provincia di Parma, che hanno raggiunto oggi il numero di 10 (più due Centri privati convenzionati), per bacino di utenza di circa 400000 abitanti, ha offerto praticamente la dialisi sotto casa alla maggior parte dei pazienti salvaguardandone le possibilità di riabilitazione e nel contempo l'equilibrio familiare. Questa scelta ha anche limitato lo sviluppo della dialisi peritoneale che in altre sedi ha rappresentato l'evoluzione naturale della dialisi domiciliare, ma dimostra oggi i suoi limiti, derivanti dall'impossibilità di seguire in strutture a basso costo assistenziale pazienti clinicamente sempre più complicati.

\section{Qual è il ruolo attuale della dialisi} domiciliare extracorporea?

Prescindendo da problemi tecnici derivanti dalla necessità di disporre di apparecchiature semplici, affidabili, di scarsa manutenzione ma non per questo meno efficaci di quelle usate oggi nei Centri dialisi, prescindendo dalla necessità di un sistema di trattamento dell'acqua di rete efficace, sicuro e facilmente controllabile, problemi tutti che potranno essere superati dalla tecnologia, emerge come aspetto principale del problema il rapporto costo/beneficio per il paziente di un trattamento completamente autogestito. Non credo che non si possa essere d'accordo sulla centralità del benessere del paziente nella scelta del trattamento sostitutivo, ma ritengo che sia difficile trovare il giusto punto di equilibrio tra il garantismo assistenziale, in cui il paziente diventa soggetto passivo della terapia e l'obbligo alla scelta prioritaria di terapie sostitutive meno costose ma che coinvolgono anche i familiari del paziente in modo da modificare profondamente le loro abitudini di vita. Infatti, a differenza di altri trattamenti domiciliari come la dialisi peritoneale, l'emodialisi richiede almeno fino a oggi una sorveglianza più attenta da parte di un partner, a causa della circolazione extracorporea. Proprio la necessità di un'assistenza domiciliare ha limitato la diffusione della CAPD nei pazienti non completamente autosufficienti nella gestione del trattamento, a favore della APD che può essere gestita con l'aiuto familiare (valido) alla fine della giornata lavorativa, con un impegno accettabile, in quanto compatibile col riposo notturno. Il numero sempre più ridotto dei pazienti autosufficienti limiterebbe quindi l'emodialisi domiciliare a una ristretta minoranza. Da una valutazione approssimativa solo 1'8\% dei pazienti trattati nel Centro ospedaliero di Parma avrebbe i requisiti clinici teorici per affrontare l'emodialisi domiciliare, senza tener conto della possibilità o meno di collaborazione di un partner. I candidati ideali sono i giovani in attesa di trapianto che, per altro, possono essere trattati adeguatamente, come prima scelta, con la dialisi peritoneale. L'emodialisi domiciliare potrebbe essere per loro una seconda scelta temporanea, mentre potrebbe essere la scelta definitiva, in alternativa alla dialisi peritoneale, per pazienti meno giovani ma autosufficienti, non candidati al trapianto renale, dopo accertamento da parte dello psicologo di una situazione familiare favorevole. Sarebbe opportuno comunque prevedere la possibilità di trasferimenti periodici nei Centri ad assistenza limitata per evitare eccessive tensioni psicologiche familiari nel trattamento a lungo termine. Il rapporto costo/beneficio del trattamento dovrebbe essere reso più favo- 
revole con l'aggiunta dei vantaggi clinici in termini di stabilità emodinamica, controllo dell'ipertensione e più efficace depurazione che il trattamento quotidiano ha dimostrato di offrire. Il vantaggio dell'abbattimento dei costi diretti e indiretti ottenibile con la maggiore diffusione dell'emodialisi domiciliare è a mio avviso di scarso rilievo dati i numeri in gioco, salvo realtà locali diverse per caratteristiche dei pazienti trattati. Infatti il problema principale della dialisi è oggi rappresentato dalla gestione del numero sempre crescente dei pazienti anziani, non autosufficienti, spesso privi di partner validi, con patologie multiple, non più trattabili in Centri dove la presenza del medico non è costante, per i quali devono essere previste delle strutture di livello intermedio tra quelle ad assistenza limitata e i Centri ospedalieri.

L'emodialisi domiciliare, inserita in una strategia terapeutica che preveda il precoce coinvolgimento del paziente e la flessibilità delle opzioni terapeutiche, resta sicuramente una valida alternativa, utile per decongestionare le strutture centralizzate, purché non venga vissuta dal paziente stesso come una imposizione senza vie d'uscita.

\section{davidren@unipr.it}

